\title{
microRNA expression profiling in multidrug resistance of the 5-Fu-induced SGC-7901 human gastric cancer cell line
}

\author{
YAN WANG $^{1 *}$, XIAODONG GU ${ }^{1 *},{\text { ZHENYANG } \text { LI }^{1}, \text { JIANBIN XIANG }^{1}, \text { JIANHAI JIANG }}^{2}$ and ZONGYOU CHEN $^{1}$ \\ ${ }^{1}$ Department of General Surgery, Huashan Hospital, Fudan University, Shanghai 200040; \\ ${ }^{2}$ Key Laboratory of Glycoconjugate Research, Ministry of Public Health and Gene Research Center, \\ Shanghai Medical College of Fudan University, Shanghai 200032, P.R. China
}

Received October 29, 2012; Accepted March 13, 2013

DOI: $10.3892 / \mathrm{mmr} .2013 .1384$

\begin{abstract}
The present study aimed to identify microRNA (miRNA) expression profiles associated with multidrug resistance (MDR) in gastric carcinoma. A 5-fluorouracil (5-Fu)-induced MDR gastric cancer cell line was selected and miRNA expression profiling of the cell line was conducted following exposure to 5-Fu. The miRNA expression profiles between the parental and resistant gastric cancer cells were analyzed by Human miRNA OneArray ${ }^{\circledR}$ v3 and the results were confirmed by quantitative real-time RT-PCR. The expression of 9 miRNAs (miR-10b, -22, -31, -133b, -190, -501, -615, $-501-5 p$ and $-615-5 p$ ) was upregulated while the expression of 18 additional miRNAs (miR-32, -197, -210, -766, -1229, -1238, $-3131,-3149,-1224-3 p,-3162-3 p,-532,-877,-4701-5 p,-5096$, $-4728-3 p,-1273 d,-486-3 p$ and $-4763-3 p)$ was downregulated in the SGC-7901/5-Fu cell line compared with its parental cell line. The results indicate that miRNA expression correlates with MDR in gastric cancer and may serve as biomolecular targets for MDR elimination.
\end{abstract}

\section{Introduction}

Gastric carcinoma is the third most common malignancy in China (1) and the second most common cause of cancer mortality, with 934,000 newly diagnosed cases and 700,000 mortalities annually worldwide (2). Owing to its vague and non-specific symptoms, gastric cancer is often diagnosed at advanced stages, with a 5-year survival rate of $<30 \%$. In addition, gastric carcinoma is associated with a high risk of recurrence even following radical surgery (3). The standard treatment for advanced or relapsed gastric cancer is chemotherapy. However, the devel-

Correspondence to: Professor Zongyou Chen, Department of General Surgery, Huashan Hospital, Fudan University, 12 Wulumuqi Middle Road, Shanghai 200040, P.R. China

E-mail: zongyouc@sohu.com

*Contributed equally

Key words: multidrug resistance, gastric cancer, microRNA opment of multidrug resistance (MDR) in gastric cancer is a substantial obstacle for chemotherapy. MDR is considered to be the main cause of failure in cancer chemotherapy and the mechanism behind it remains unclear.

Accumulating evidence has suggested that microRNAs (miRNAs) are key players in MDR. The miRNAs are a large family of small non-coding RNAs of 19-25 nucleotides that negatively control gene expression at the mRNA and protein level. Microarray studies have revealed that individual miRNAs affect the expression of multiple genes, indicating that miRNAs have pleiotropic effects in crucial biological processes, including development, differentiation and apoptosis $(4,5)$. Since a number of biological processes are relevant to chemosensitivity and chemoresistance, miRNA is thought to be associated with drug resistance in cancer, as demonstrated in several previous studies (6-8). In addition, treatment with antineoplastic agents alters the expression of miRNAs in vitro and in vivo $(9,10)$. However, few studies on MDR-related miRNAs in gastric carcinoma have been performed.

The aim of the present study was to understand the molecular mechanism of MDR in human gastric cancer cells following exposure to 5-fluorouracil (5-Fu). A 5-Fu-induced MDR gastric cancer cell line was developed and microarray analysis was performed to directly characterize the molecular basis of MDR. Following this, the miRNA expression profiles between the MDR and the parental cell lines were compared.

\section{Materials and methods}

Cell lines and cell culture. The human gastric cancer cell line, SGC-790, was purchased from the Shanghai Institutes for Biological Sciences, Chinese Academy of Sciences (Shanghai, China). SGC-7901 was maintained in RPMI-1640 medium (Hyclone, Logan, UT, USA) supplemented with $10 \%$ fetal bovine serum (Hyclone). The cells were removed from the surface of the culture vessels by trypsinization and then washed with phosphate-buffered saline (PBS). Aliquots $(0.5 \mathrm{ml})$ of the cell suspension were added to $4.5 \mathrm{ml}$ fresh medium in $30-\mathrm{cm}^{2}$ flasks. This study was approved by the ethics committee of Huashan Hospital of Fudan University, Shanghai, China.

Establishment of the drug-resistant cell line, SGC-7901/5-Fu, in vitro. The resistant cell line was produced by continuous 
exposure to 5-Fu (Sigma, St. Louis, MO, USA) of low and gradually increasing concentrations. When the cells were growing exponentially, $5 \mu \mathrm{g} / \mathrm{ml} 5$-Fu was added to the medium. Following $48 \mathrm{~h}$ of incubation, the majority of the cells were dead. The treated cells were then washed with PBS and cultured in 5-Fu free growth medium. Following 2-3 days, the dead cells were washed away with PBS and fresh medium was added. The resistant sub-clones were isolated by limiting dilution. The cells were passaged at $80 \%$ confluency and then $5 \mu \mathrm{g} / \mathrm{ml} 5$-Fu was added. The concentration of 5-Fu was gradually increased following stable cell growth. Finally, a cell line resistant to $40 \mu \mathrm{g} / \mathrm{ml} 5$-Fu was derived from the SGC-7901 cell line. Prior to further experimentation, these cells were maintained in a 5-Fu-free culture medium for 1 month and subcultured at least 3 times.

In vitro drug sensitivity assay. Cisplatin (CDDP), vincristine (VCR) and adriamycin (ADM) were all purchased from Sigma. The 5-Fu, CDDP, VCR and ADM were all freshly prepared prior to each experiment. The drug sensitivity was evaluated by the 3-(4,5-dimethylthiazol-2-yl)-2,5-diphenyltetrazolium bromide (MTT) colorimetric assay. Following this, the cells were cultured in 96-well microculture plates in the presence or absence of various concentrations of the drugs for $48 \mathrm{~h}$. A $0.05 \mathrm{ml}$ MTT solution (0.1\%) in PBS was added to each well followed by incubation for an additional $4 \mathrm{~h}$. The medium was removed and the purple formazan product in the cells was measured at a wavelength of $540 \mathrm{~nm}$ and the number of viable cells was calculated. Measurements were performed in triplicate and dose-response curves were plotted using data derived from the MTT assay. The half maximal inhibitory concentration $\left(\mathrm{IC}_{50}\right)$ of each anticancer drug was calculated from this standard curve.

Western blot analysis. Protein extracts of the SGC-7901 and SGC-7901/5-Fu cells were resolved by $12 \%$ sodium dodecyl sulfate-polyacrylamide gel electrophoresis (SDS-PAGE) and transferred to polyvinylidene difluoride (PVDF) membranes (Millipore, Billerica, MA, USA). Subsequent to blocking, the PVDF membranes were washed three times for $15 \mathrm{~min}$ with Tris-Buffered Saline with Tween-20 (TBST) at room temperature and incubated with mouse anti-human P-glycoprotein (eBioscience, San Diego, CA, USA). P-glycoprotein is also known as multidrug resistance protein 1 (MDR1). Following extensive washing, the membranes were incubated with horseradish peroxidase-linked goat anti-mouse IgG (Santa Cruz Biotechnology, Santa Cruz, CA, USA) for $1 \mathrm{~h}$. Following an additional TBST wash, the immunoreactivity was visualized using an electrogenerated chemiluminescence kit (Pierce Biotechnology, Rockford, IL, USA) according to the manufacturer's instructions.

miRNA microarray. Total RNA was extracted using TRIzol reagent (Invitrogen Life Technologies, Carlsbad, CA, USA) as described previously (11). RNA labeling and hybridization to the miRNA microarray chips were performed according to the manufacturer's instructions. Briefly, $2.5 \mu \mathrm{g}$ RNA from each sample was labeled with the miRNA Universal Linkage System (ULS) ${ }^{\mathrm{TM}}$ Labeling kit (Kreatech Diagnostics, Amsterdam, Netherlands). Hybridization was performed
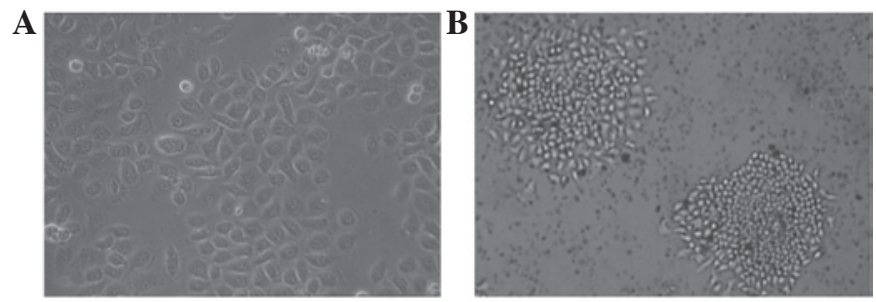

Figure 1. Cell shape of the (A) SGC-7901 and (B) SGC-7901/5-Fu cell lines 5-Fu, 5-fluorouracil; SGC-7901, human gastric adenocarcinoma cell line with an epithelial-like morphology; SGC-7901/5-Fu, 5-Fu-resistant cell line from the parental cell line. Magnification, (A) x40 and (B) x10.

on the Human miRNA OneArray ${ }^{\circledR}$ v3 (Phalanx Biotech Group, Belmont, CA, USA), which contains 1,711 human and 189 experimental control probes. There were three spot replicates for each probe. Hybridization images were captured using a GenePix 4000B laser scanner (Molecular Devices, Sunnyvale, CA, USA) and the data was analyzed by GenePix Pro 6.0 software (Molecular Devices).

Quantitative real-time RT-PCR of miRNA and $m R N A$. The expression of the miRNAs and the drug resistance gene, MDR1, was assessed using quantitative real-time RT-PCR as described previously (12). cDNA synthesis was performed using the miScript Reverse Transcription kit (Qiagen, Hilden, Germany) according to the manufacturer's instructions. Quantitative PCR was performed using the miScript SYBR Green PCR kit (Qiagen, Hilden, Germany). An expression analysis was performed in triplicate for each sample. The small nuclear RNA, U6, was used as the normalization control. The miRNA and mRNA expression levels were quantified using the ABI 7300 Sequence Detection System (Applied Biosystems, Foster City, CA, USA). Three independent experiments were performed in triplicate.

Statistical analysis. All data are presented as the mean \pm SD. Statistical analyses were performed using Stata 8.0 software (Stata, College Station, TX, USA). The comparison of the $\mathrm{IC}_{50}$ and the miRNA and MDR1 expression between the MDR and parental cell lines were tested by the Student's t-test. $\mathrm{P}<0.05$ was considered to indicate a statistically significant difference. Data was normalized and the differentially expressed miRNAs were processed using GenePix Pro 6.0 software.

\section{Results}

Establishment of a 5-Fu-resistant SGC-7901/5-Fu cell line. The 5-Fu-resistant SGC-7901 (SGC-7901/5-Fu) cell line was established by pulse exposure of the SGC-7901 cells to gradually increasing concentrations of 5-Fu. This led to a marked alteration in cellular morphology. The parental cells (Fig. 1A) appeared as small, round or polygonal cells forming a 'cobblestone' arrangement, with a doubling time of $16.4 \mathrm{~h}$. The cells revealed little contact inhibition and tended to grow in piled-up clusters. Following acquisition of 5-Fu resistance, the doubling time of the SGC-7901/5-Fu cells (Fig. 1B) was slightly longer at $22.1 \mathrm{~h}$ and the cells exhibited notable morphological changes characterized by transition from an epithelial cobblestone to an elongated fibroblastic phenotype. 
Table I. IC S0 $_{5}$ and RI values of the SGC-7901 and SGC-7901/5-Fu cells.

\begin{tabular}{lccc}
\hline & \multicolumn{2}{c}{$\mathrm{IC}_{50}$, mean $\pm \mathrm{SD}$} & \\
\cline { 2 - 3 } Drug $(\mu \mathrm{g} / \mathrm{ml})$ & SGC-7901 & SGC-7901/5-Fu & RI \\
\hline 5 -Fu & $5.0 \pm 0.02$ & $155.0 \pm 3.1^{\mathrm{a}}$ & 31.0 \\
CDDP & $3.6 \pm 0.12$ & $10.7 \pm 0.31^{\mathrm{a}}$ & 2.97 \\
VCR & $1.3 \pm 0.11$ & $3.3 \pm 0.12^{\mathrm{a}}$ & 2.54 \\
ADM & $0.57 \pm 0.06$ & $3.53 \pm 0.15^{\mathrm{a}}$ & 6.19
\end{tabular}

5-Fu, 5-fluorouracil; CDDP, cisplatin; VCR, vincristine; ADM, adriamycin; $\mathrm{IC}_{50}$, drug concentration $(\mu \mathrm{g} / \mathrm{ml})$ at which cell growth is inhibited by $50 \%$; RI, resistance index $\left(\mathrm{IC}_{50}\right.$ for resistant cell line/ $\mathrm{IC}_{50}$ for parental cell line). The values of 3 independent experiments are presented as mean $\pm \mathrm{SD}$. ${ }^{\mathrm{a}} \mathrm{P}<0.05$, vs. parental cells.

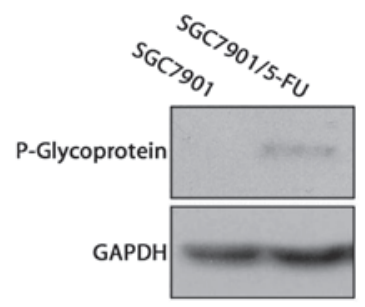

Figure 2. Western blot analysis of P-glycoprotein in SGC-7901 and SGC-7901/5-Fu cells. Data are representative of those obtained in 3 other experiments. 5-Fu, 5-fluorouracil; GAPDH, glyceraldehyde 3-phosphate dehydrogenase.

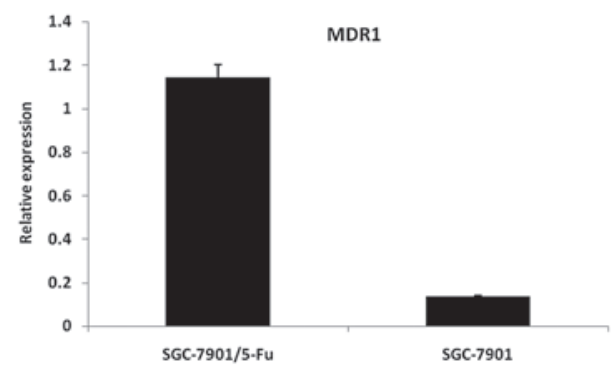

Figure 3. Expression of MDR1 mRNA in SGC-7901 and SGC-7901/5-Fu cells. Data are presented as mean $\pm \mathrm{SD}$ from three independent experiments. MDR1, multidrug resistance protein 1; 5-Fu, 5-fluorouracil..

The resistant cells became smaller in size and tended to form well-separated individual colonies.

The sensitivity of the SGC-7901 and SGC-7901/5-Fu cells to various concentrations of $5-\mathrm{Fu}$ was determined by MTT assay. As demonstrated in Table I, the $\mathrm{IC}_{50}$ values for $5-\mathrm{Fu}$ in the SGC-7901 and SGC-7901/5-Fu cells were 5.0 \pm 0.02 and $155.0 \pm 3.1 \mu \mathrm{g} / \mathrm{ml}$, respectively. The SGC-7901/5-Fu cells were 31-fold more resistant to 5-Fu than the parent cells. In addition, the cross-resistance to other anticancer drugs (CDDP, VCR, ADM) was compared between the parent and 5-Fu-resistant cells and the results indicated that the SGC-7901/5-Fu cells also had cross-resistance to CDDP, VCR and ADM.
Table II. miRNAs correlated with multidrug resistance of the SGC-7901/5-Fu cell line.

\begin{tabular}{lcc}
\hline miRNA & $\begin{array}{c}\text { Regulation in } \\
\text { SGC-7901/5-Fu }\end{array}$ & $\begin{array}{c}\text { Chromosomal } \\
\text { location }\end{array}$ \\
\hline miR-10b & Up & 2 \\
miR-22 & Up & 17 \\
miR-31 & Up & 9 \\
miR-32 & Down & 9 \\
miR-133b & Up & 6 \\
miR-190 & Up & 15 \\
miR-197 & Down & 1 \\
miR-210 & Down \\
miR-486-3p & Down & 11 \\
miR-501 & Up & 14 \\
miR-532 & Down & X \\
miR-615 & Up & X \\
miR-501-5p & Up & 12 \\
miR-615-5p & Up & X \\
miR-766 & Down & 12 \\
miR-877 & Down & X \\
miR-1224-3p & Down & 6 \\
miR-1229 & Down & 3 \\
miR-1238 & Down & 5 \\
miR-1273d & Down & 19 \\
miR-3131 & Down & 15 \\
miR-3149 & Down & 2 \\
miR-3162-3p & Down & 8 \\
miR-4701-5p & Down \\
miR-4728-3p & Down & 11 \\
miR-4763-3p & Down & 12 \\
miR-5096 & Down & 17 \\
\hline & & 22 \\
& & 4 \\
\hline
\end{tabular}

5-Fu, 5-fluorouracil; miRNA, microRNA.

Western blot analysis of P-glycoprotein expression. The P-glycoprotein expression in the SGC-7901/5-Fu cells was evaluated by western blot analysis. The protein was detected in the parental SGC-7901 and SGC-7901/5-Fu cells. The expression levels of the P-glycoprotein were significantly higher in the SGC-7901/5-Fu-resistant cells than in the SGC-7901 cells (Fig. 2). Confirmation of the maintenance of P-glycoprotein expression and its functionality were important for the culture conditions.

Evaluation of MDRI $m R N A$ by quantitative real-time $R T-P C R$. The expression of the MDR1 mRNA was examined by quantitative real-time RT-PCR and the results are presented in Fig. 3. The 5-Fu-resistant cells demonstrated increased levels of MDR1 mRNA expression when compared with the SGC-7901 cells $(\mathrm{P}<0.05)$.

miRNA expression profiling in the $S G C-7901$ vs. $S G C-7901 / 5-F u$ cell lines. To investigate the role of miRNAs in the MDR of human gastric cancer cells following expo- 


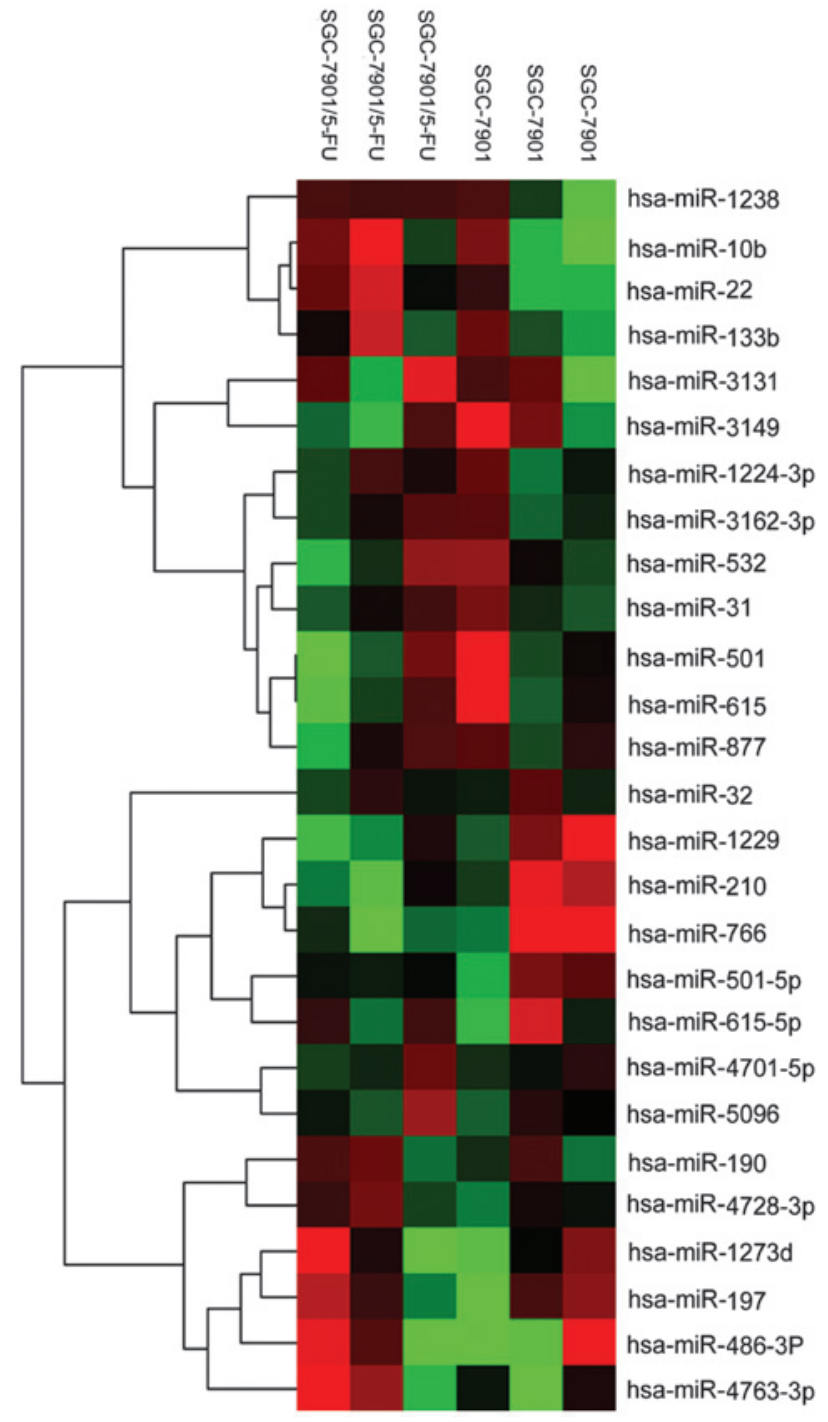

Figure 4. miRNA expression signature in SGC-7901 vs. SGC-7901/5-Fu cells. The heatmap is based on 27 differentially expressed miRNAs. Red, upregulated; green, downregulated; black, no change; 5-Fu, 5-fluorouracil; miRNA, microRNA; hsa, Homo sapiens.

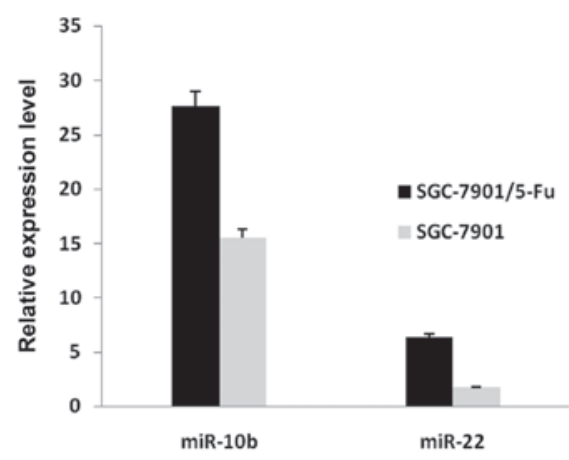

Figure 5. Validation of microarray data using real-time RT-PCR. Analysis of miR-10b and miR-22 was performed to validate the microarray results. Data are presented as mean $\pm \mathrm{SD}$ from three independent experiments. 5-Fu, 5-fluorouracil; miRNA, microRNA.

sure to $5-\mathrm{Fu}$, comprehensive miRNA expression profiling of SGC-7901 and SGC-7901/5-Fu cells was conducted using the Human miRNA OneArray ${ }^{\circledR}$ v3. The results revealed that 18 miRNAs were downregulated $>2$-fold in the SGC-7901/5-Fu cells compared with the SGC-7901 cells, while 9 miRNAs were upregulated $>2$-fold in the SGC-7901/5-Fu cells (Table II). These results indicate that these 27 miRNAs may play significant roles in the development of MDR when treating human gastric cancer cells with 5-Fu. In addition, a hierarchical cluster analysis based on the expression patterns of these 27 miRNAs accurately separated the SGC-7901/5-Fu cells from the SGC-7901 cells (Fig. 4).

Validation of microarray data by real-time RT-PCR. To confirm the results of the miRNA microarray analysis, miR-10b and miR-22 were selected for analysis by real-time quantitative RT-PCR. The real-time RT-PCR results were consistent with the miRNA microarray analysis results (Fig. 5).

\section{Discussion}

In the present study, the MDR gastric cell line took 8 months to be successfully established. In addition, the primary colonies were dissociated into single cells and then cultured. A single cell formed a secondary colony. The secondary colonies generated tertiary colonies, indicating that the colony-forming cells isolated from the existing colonies retained the same colony-forming potential. These observations indicated that the resistant cells had a self-renewal capacity resembling that of cancer stem cells; this was reported for the first time in the present study.

MDR is defined as the resistance of tumor cells to a variety of structurally dissimilar and functionally divergent chemotherapeutic agents. MDR mediates the process of drug inactivation and removal from the target tumor cells (13) and is a major obstacle for the treatment of cancer. Although the mechanism of MDR remains unclear, it has been hypothesized that MDR is a multifactorial process which includes the following four events: i) the altered expression of membrane transport proteins to increase drug efflux, ii) enhanced DNA repair, iii) the amendment of cell cycle regulation to block apoptosis and iv) detoxification through increasing drug metabolism and decreasing drug activation (14).

The first mechanism to be associated with MDR was identified in 1988 (15,16). P-glycoprotein, an active, energy dependent multidrug transporter in the membrane, was identified and reported to impede intracellular cytotoxic drug accumulation by rapid extrusion. In the present study, SGC-7901/5-Fu cells were also identified to overexpress P-glycoprotein and MDR1 mRNA. However, a previous study reported that in specific P-glycoprotein-negative cancer cell lines, the resistance to a wide variety of chemotherapy drugs was observed (17). These data indicate that there may be additional factors which are important in MDR (18).

miRNAs are vital to a number of biological processes, including development, proliferation and apoptosis (19). A single miRNA may target dozens of mRNAs and the dysregulation of miRNAs affects the expression of multiple target mRNAs and therefore multiple proteins, leading to variations in the chemotherapy sensitivity/resistance to common cancer treatments via various cellular processes. Previously, a correlation was identified between miRNA expression and chemoresistance in several types of cancer. The expression 
of miR-34a attenuated the chemoresistance to camptothecin in prostate cancer cells (20). The dysregulation of let-7i was also reported to be significantly correlated with chemoresistance in ovarian and breast cancer (21). In glioblastoma cells, miR-21 has been reported to contribute to teniposide resistance (22).

However, few studies have determined the potential role of miRNAs in the chemoresistance of gastric cancer. The present study is the first to perform miRNA expression profiling in the MDR human gastric cancer cell line, SGC-7901, following exposure to $5-\mathrm{Fu}$. The results indicate that the expression of 9 miRNAs (miR-10b, -22, -31, -133b, -190, -501, -615, -501-5p and $-615-5 \mathrm{p}$ ) was upregulated, while 18 miRNAs (miR-32, $-197,-210,-766,-1229,-1238,-3131,-3149,-1224-3 p,-3162-3 p$, $-532,-877,-4701-5 p,-5096,-4728-3 p,-1273 d,-486-3 p$ and $-4763-3 p)$ were downregulated in the SGC-7901/5-Fu cell line compared with its parental cell line. In comparison to previous studies, the miRNA expression profile in the SGC-7901/VCR cell line was completely different to that of the SGC-7901/5-Fu cell line (23), indicating that each antineoplastic agent is able to alter the expression of relevant miRNAs.

The quantitative RT-PCR analysis of miR-10b and miR-22 confirmed the expression patterns identified by microarray. Numerous previous studies $(24,25)$ have found that miR-10b was highly expressed in metastatic breast cancer cells and that it regulated cell migration and invasion. In addition, miR-10b has been identified to positively regulate the metastasis of nasopharyngeal carcinoma (26). Considering the flexible functions of miR-10b involved in biological processes closely associated with chemoresistance, including development, apoptosis and proliferation, the miRNA molecule is believed to be correlated with drug resistance in cancer. In addition, a previous study reported that curcumin and piperine, individually and in combination, were able to inhibit breast cancer stem cell self-renewal by the upregulation of miR-22, leading to the restoration of the susceptibility to chemotherapy (27).

In summary, in the present study, 27 differentially expressed miRNAs were identified between the parental and resistant gastric cancer cells. The acquisition of MDR by the cells indicated that miRNAs are key to the anticancer drug response. These observations are the first to show that resistant cells have a self-renewal capability and colony-forming potential resembling that of cancer stem cells in gastric cancer chemotherapy. The results provide a novel insight into the mechanisms of MDR in gastric cancer and may be useful for the future development of a chemosensitizing strategy through the manipulation of miRNA expression.

\section{Acknowledgements}

The present study was supported by the Shanghai Young Doctor Training Plan and a grant from the Major Research and Development Project of Innovative Drugs, Ministry of Science and Technology (no. 2012ZX09303004-001).

\section{References}

1. Zhao P, Dai M, Chen W and Li N: Cancer trends in China. Jpn J Clin Oncol 40: 281-285, 2010.
2. Jemal A, Siegel R, Ward E, et al: Cancer statistics, 2008. CA Cancer J Clin 58: 71-96, 2008.

3. de Bree E, Charalampakis V, Melissas J and Tsiftsis DD: The extent of lymph node dissection for gastric cancer: a critical appraisal. J Surg Oncol 102: 552-562, 2010.

4. Lagos-Quintana M, Rauhut R, Lendeckel W and Tuschl T: Identification of novel genes coding for small expressed RNAs. Science $294: 853-858,2001$

5. Cheng AM, Byrom MW, Shelton J and Ford LP: Antisense inhibition of human miRNAs and indications for an involvement of miRNA in cell growth and apoptosis. Nucleic Acids Res 33: 1290-1297, 2005.

6. Blower PE, Chung JH, Verducci JS, et al: MicroRNAs modulate the chemosensitivity of tumor cells. Mol Cancer Ther 7: 1-9, 2008.

7. Zheng T, Wang J, Chen X and Liu L: Role of microRNA in anticancer drug resistance. Int J Cancer 126: 2-10, 2010.

8. Sarkar FH, Li Y, Wang Z, Kong D and Ali S: Implication of microRNAs in drug resistance for designing novel cancer therapy. Drug Resist Updat 13: 57-66, 2010.

9. Rossi L, Bonmassar E and Faraoni I: Modification of miR gene expression pattern in human colon cancer cells following exposure to 5-fluorouracil in vitro. Pharmacol Res 56: 248-253, 2007.

10. Nakajima G, Hayashi K, Xi Y, et al: Non-coding microRNAs hsa-let-7g and hsa-miR-181b are associated with chemoresponse to S-1 in colon cancer. Cancer Genomics Proteomics 3: 317-324, 2006.

11. Rosenfeld N, Aharonov R, Meiri E, et al: MicroRNAs accurately identify cancer tissue origin. Nat Biotechnol 26: 462-469, 2008.

12. Schmittgen TD, Lee EJ, Jiang J, et al: Real-time PCR quantification of precursor and mature microRNA. Methods 44: 31-38, 2008.

13. Luqmani YA: Mechanisms of drug resistance in cancer chemotherapy. Med Princ Pract 14 (Suppl 1): 35-48, 2005.

14. Ganguly A, Banerjee K, Chakraborty P, et al: Overcoming multidrug resistance (MDR) in cancer in vitro and in vivo by a quinoline derivative. Biomed Pharmacother 65: 387-394, 2011.

15. Gottesman MM and Pastan I: The multidrug transporter, a double-edged sword. J Biol Chem 263: 1263-1266, 1988.

16. Moscow JA and Cowan KH: Multidrug resistance. J Natl Cancer Inst 80: 14-20, 1988

17. Watson MB, Lind MJ and Cawkwell L: Establishment of in-vitro models of chemotherapy resistance. Anticancer Drugs 18: 749-754, 2007.

18. Singh R and Lillard JW Jr: Nanoparticle-based targeted drug delivery. Exp Mol Pathol 86: 215-223, 2009.

19. Hammond SM, Bernstein E, Beach D and Hannon GJ: An RNA-directed nuclease mediates post-transcriptional gene silencing in Drosophila cells. Nature 404: 293-296, 2000.

20. Fujita Y, Kojima K, Hamada N, et al: Effects of miR-34a on cell growth and chemoresistance in prostate cancer PC3 cells. Biochem Biophys Res Commun 377: 114-119, 2008.

21. Northcott PA, Fernandez-L A, Hagan JP, et al: The miR-17/92 polycistron is up-regulated in sonic hedgehog-driven medulloblastomas and induced by $\mathrm{N}$-myc in sonic hedgehog-treated cerebellar neural precursors. Cancer Res 69: 3249-3255, 2009.

22. Li Y, Li W, Yang Y, et al: MicroRNA-21 targets LRRFIP1 and contributes to VM-26 resistance in glioblastoma multiforme. Brain Res 1286: 13-18, 2009.

23. Xia L, Zhang D, Du R, et al: miR-15b and miR-16 modulate multidrug resistance by targeting BCL2 in human gastric cancer cells. Int J Cancer 123: 372-379, 2008.

24. Ma L, Teruya-Feldstein J and Weinberg RA: Tumour invasion and metastasis initiated by microRNA-10b in breast cancer. Nature 449: 682-688, 2007.

25. Gee HE, Camps C, Buffa FM, et al: MicroRNA-10b and breast cancer metastasis. Nature 455: E8-E9, 2008.

26. Li G, Wu Z, Peng Y, et al: MicroRNA-10b induced by Epstein-Barr virus-encoded latent membrane protein-1 promotes the metastasis of human nasopharyngeal carcinoma cells. Cancer Lett 299: 29-36, 2010.

27. Kakarala M, Brenner DE, Korkaya H, et al: Targeting breast stem cells with the cancer preventive compounds curcumin and piperine. Breast Cancer Res Treat 122: 777-785, 2010. 